\title{
LOCAL ASYMPTOTIC NORMALITY PROPERTY FOR FRACTIONAL GAUSSIAN NOISE UNDER HIGH-FREQUENCY OBSERVATIONS
}

\author{
By AleXANDre Brouste* AND MASAAKI FuKASAWA ${ }^{\dagger} \neq$ \\ Le Mans Université*, Osaka University ${ }^{\dagger}$ and Tokyo Metropolitan University
}

\begin{abstract}
Local Asymptotic Normality (LAN) property for fractional Gaussian noise under high-frequency observations is proved with nondiagonal rate matrices depending on the parameter to be estimated. In contrast to the LAN families in the literature, nondiagonal rate matrices are inevitable. As consequences of the LAN property, a maximum likelihood sequence of estimators is shown to be asymptotically efficient and the likelihood ratio test on the Hurst parameter is shown to be an asymptotically uniformly most powerful unbiased test for two-sided hypotheses.
\end{abstract}

1. Introduction. The theory of Local Asymptotic Normality (LAN) provides a powerful framework under which we are able to discuss asymptotic optimality of estimators. When the LAN property holds true for a statistical experiment, minimax theorems $[13,20]$ can be applied and a lower bound for the variance of the estimators can be derived. Beyond the classical i.i.d. setting [14, 26], the LAN property (or Local Asymptotic Mixed Normality property) has been proved for various statistical models including linear processes [25], ergodic Markov chains [24], ergodic diffusions [11, 19], diffusions under high-frequency observations [12], diffusions with observational noise $[9,10,23]$ and several Lévy process models $[4$, $17,18]$. The LAN property for fractional Gaussian noise (fGn) was obtained in [6] under the large sample observation scheme. In this setting, Maximum Likelihood (ML) and Whittle sequences of estimators achieve optimality [7, 8, 21].

The statistical experiment of observing fGn under a high-frequency scheme has not been well understood in the literature, despite that high-frequency data has attracted much attention recently due to their increasing availability. At high frequency, scaling effects from the variance and from the self-similarity of the fGn are melting. The singularity of the joint estimation of diffusion coefficient and Hurst parameter was already noticed in $[2,5,15]$. A weak LAN property with a singular Fisher matrix was obtained in [16]. Due to this singularity, no minimax theorem can be applied and in particular, it has been unclear whether the ML estimator enjoys any kind of optimality property.

Received October 2016; revised February 2017.

MSC2010 subject classifications. 62F05, 62F12.

Key words and phrases. Locally asymptotically normal families, fractional Brownian motion, high-frequency data, maximum likelihood estimators, likelihood ratio test. 
In this paper, we prove that the statistical experiment in fact enjoys the LAN property. To discuss the difference from [16], let us be more precise in the definition of the LAN property.

Definition 1.1. Let $\Theta \subset \mathbb{R}^{d}$. A family of measures $\left\{P_{\theta}^{n}, \theta \in \Theta\right\}$ is called locally asymptotically normal (LAN) at $\theta_{0} \in \Theta$ if there exist nondegenerate $d \times d$ matrices $\varphi_{n}\left(\theta_{0}\right)$ and $I\left(\theta_{0}\right)$ such that for any $u \in \mathbb{R}^{d}$, the likelihood ratio

$$
Z_{n}(u)=\frac{\mathrm{d} P_{\theta_{0}+\varphi_{n}}^{n}\left(\theta_{0}\right) u}{\mathrm{~d} P_{\theta_{0}}^{n}}
$$

admits the representation

$$
Z_{n}(u)=\exp \left(\left\langle u, \zeta_{n}\left(\theta_{0}\right)\right\rangle-\frac{1}{2}\left\langle I\left(\theta_{0}\right) u, u\right\rangle+r_{n}\left(\theta_{0}, u\right)\right),
$$

where

$$
\zeta_{n}\left(\theta_{0}\right) \rightarrow \mathcal{N}\left(0, I\left(\theta_{0}\right)\right), \quad r_{n}\left(\theta_{0}, u\right) \rightarrow 0
$$

in law under $P_{\theta_{0}}^{n}$.

This definition of the LAN property is equivalent to the one given in [14]. The matrix $\varphi_{n}\left(\theta_{0}\right)$ is often called the rate matrix. Remark that the nondegeneracy of $\varphi_{n}\left(\theta_{0}\right)$ and $I\left(\theta_{0}\right)$ is essential in the following minimax theorem due to Hajek [13] and Le Cam [20], which implies in particular the asymptotic efficiency of the ML sequence of estimators in regular models; see, for example, Theorem II.12.1 and Remark II.12.2 in [14].

THEOREM 1.1. Let the family of measures $\left\{P_{\theta}^{n}, \theta \in \Theta\right\}, \Theta \subset \mathbb{R}^{d}$, be LAN at $\theta_{0} \in \Theta$ for nondegenerate matrices $\varphi_{n}\left(\theta_{0}\right)$ and $I\left(\theta_{0}\right)$. Then, for any sequence of estimators $\hat{\theta}_{n}$,

$$
\begin{gathered}
\liminf _{\delta \rightarrow \infty} \liminf _{n \rightarrow \infty} \sup _{\left|\varphi_{n}\left(\theta_{0}\right)^{-1}\left(\theta-\theta_{0}\right)\right| \leq \delta} E_{\theta}^{n}\left[l\left(\varphi_{n}\left(\theta_{0}\right)^{-1}\left(\hat{\theta}_{n}-\theta\right)\right)\right] \\
\geq \int_{\mathbb{R}^{d}} l\left(I\left(\theta_{0}\right)^{-1 / 2} z\right) \phi(z) \mathrm{d} z
\end{gathered}
$$

for any symmetric, nonnegative quasi-convex function $l$ with

$$
\lim _{|z| \rightarrow \infty} e^{-\varepsilon|z|^{2}} l(z)=0
$$

for all $\varepsilon>0$, where $\phi$ is the density of the d-dimensional standard normal distribution. 
For the fGn under high-frequency observations, we consider the estimation of the parameters $(H, \sigma) \in \Theta=(0,1) \times(0, \infty)$, where $H$ is the Hurst parameter and $\sigma$ is the diffusion coefficient. It was shown in [16] that the family of measures $\left\{P_{(H, \sigma)}^{n},(H, \sigma) \in \Theta\right\}$ satisfies both conditions (1) and (2) at any $(H, \sigma) \in \Theta$ for

$$
\varphi_{n}(H, \sigma)=\left(\begin{array}{cc}
\frac{1}{\sqrt{n} \log \Delta_{n}} & 0 \\
0 & \frac{1}{\sqrt{n}}
\end{array}\right) \quad \text { and } \quad I(H, \sigma)=\left(\begin{array}{cc}
2 & 2 / \sigma \\
2 / \sigma & 2 / \sigma^{2}
\end{array}\right)
$$

where $n$ is the sample size and $\Delta_{n}$ is the length of sampling intervals. Note that $I(H, \sigma)$ is singular. On the one hand, this result does not imply that the family is LAN in the sense of Definition 1.1. On the second hand, Theorem 1.1 cannot be applied with this result.

To the best of our knowledge, for any LAN family in the literature, it is always possible to take a diagonal rate matrix up to a reparametrization. A typical example is the LAN property for the i.i.d. setting where

$$
\varphi_{n}\left(\theta_{0}\right)=\frac{1}{\sqrt{n}} \mathbf{I}_{d} .
$$

Here, $\mathbf{I}_{d}$ is the $d \times d$ identity matrix. However, both in Definition 1.1 and Theorem $1.1, \varphi_{n}\left(\theta_{0}\right)$ is required only to be nondegenerate. In this paper, we prove the LAN property for the statistical experiment of observing fGn under a highfrequency scheme for a certain class of nondiagonal matrices $\varphi_{n}\left(\theta_{0}\right)$ depending on $\theta_{0}$. In particular, Theorem 1.1 can be applied. Nondiagonal rate matrices are inevitable because the Fisher matrix is singular.

Basics for the fractional Brownian motion and the fGn are recalled in Section 2. The statistical experiment under a high-frequency scheme is described and the LAN property result is proved in Section 3. Efficient rates for the estimation of $H$ and $\sigma$ are given in Section 4 giving the optimality of the ML sequence of estimators and the asymptotically uniformly most powerful unbiased property for the likelihood ratio test on the Hurst parameter for two-sided hypotheses.

2. Fractional Brownian motion and fractional Gaussian noise. Here, we briefly review the basics of the fractional Brownian motion, fractional Gaussian noise and their large sample theory. A centered Gaussian process $B^{H}$ is called a fractional Brownian motion with Hurst parameter $H$ if

$$
E\left[B_{t}^{H} B_{s}^{H}\right]=\frac{1}{2}\left(|t|^{2 H}+|s|^{2 H}-|t-s|^{2 H}\right)
$$

for all $t, s \in \mathbb{R}$. Such a process exists and is continuous for any $H \in(0,1)$ by Kolmogorov's extension and continuity theorems. The process enjoys a self-similarity property: for any $\Delta>0$ and $t \in \mathbb{R}$,

$$
B_{t+\Delta}^{H}-B_{t}^{H} \sim \Delta^{H} B_{1}^{H}
$$


in law. The spectral density of regular increments, that is, the function $f_{H}$ characterized by

$$
\begin{aligned}
E\left[B_{1}^{H}\left(B_{k+1}^{H}-B_{k}^{H}\right)\right] & =\frac{1}{2}\left(|k+1|^{2 H}-2|k|^{2 H}+|k-1|^{2 H}\right) \\
& =\frac{1}{2 \pi} \int_{-\pi}^{\pi} e^{\sqrt{-1} k \lambda} f_{H}(\lambda) \mathrm{d} \lambda, \quad k \in \mathbb{Z}
\end{aligned}
$$

is given by

$$
f_{H}(\lambda)=C_{H} 2(1-\cos (\lambda)) \sum_{k \in \mathbb{Z}}|\lambda+2 k \pi|^{-2 H-1}, \quad C_{H}=\frac{\Gamma(2 H+1) \sin (\pi H)}{2 \pi} .
$$

For a fixed interval $\Delta>0$, assume we observe the increments

$$
X_{n}=\left(\sigma B_{\Delta}^{H}, \sigma B_{2 \Delta}^{H}-\sigma B_{\Delta}^{H}, \sigma B_{3 \Delta}^{H}-\sigma B_{2 \Delta}^{H}, \ldots, \sigma B_{n \Delta}^{H}-\sigma B_{(n-1) \Delta}^{H}\right),
$$

where $(H, \sigma)$ is unknown. The random vector $X_{n}$ is called fractional Gaussian noise (fGn). Denote by $T_{n}(H)$ the covariance matrix of

$$
\frac{X_{n}}{\sigma \Delta^{H}}
$$

of which the distribution does not depend on $\sigma$ and $\Delta$ by the self-similarity property. The $(i, j)$ element of $T_{n}(H)$ is given by

$$
\frac{1}{2 \pi} \int_{-\pi}^{\pi} e^{\sqrt{-1}(i-j) \lambda} f_{H}(\lambda) \mathrm{d} \lambda .
$$

Let us suppose $\Delta=1$ for brevity. The log-likelihood of $X_{n}$ is then given by

$$
\begin{aligned}
\ell_{n}(H, \sigma)= & -\frac{n}{2} \log (2 \pi)-n \log \sigma \\
& -\frac{1}{2} \log \left|T_{n}(H)\right|-\frac{1}{2 \sigma^{2}}\left\langle X_{n}, T_{n}(H)^{-1} X_{n}\right\rangle .
\end{aligned}
$$

Let

$$
\begin{aligned}
& A_{n}=\sqrt{n}\left\{\frac{1}{n \sigma^{2}}\left\langle X_{n}, T_{n}(H)^{-1} X_{n}\right\rangle-1\right\} \\
& B_{n}=\frac{1}{\sqrt{n}}\left\{\frac{1}{2} \partial_{H} \log \left|T_{n}(H)\right|+\frac{1}{2 \sigma^{2}}\left\langle X_{n}, \partial_{H}\left\{T_{n}(H)^{-1}\right\} X_{n}\right\rangle\right\} .
\end{aligned}
$$

The score function is then given by

$$
\nabla \ell_{n}=\left(\begin{array}{c}
\partial_{H} \ell_{n} \\
\partial_{\sigma} \ell_{n}
\end{array}\right)=\left(\begin{array}{c}
-B_{n} \sqrt{n} \\
A_{n} \sqrt{n} \sigma^{-1}
\end{array}\right)
$$

Let $P_{(H, \sigma)}^{n}$ be the measure on $\mathbb{R}^{n}$ induced by $X_{n}$. 
THEOREM 2.1. The family of measures $\left\{P_{(H, \sigma)}^{n} ;(H, \sigma) \in(0,1) \times(0, \infty)\right\}$ is $L A N$ at any $(H, \sigma) \in(0,1) \times(0, \infty)$ with

$$
\varphi_{n}(H, \sigma)=\left(\begin{array}{cc}
\frac{1}{\sqrt{n}} & 0 \\
0 & \frac{1}{\sqrt{n}}
\end{array}\right)
$$

and

$$
I(H, \sigma)=\left(\begin{array}{cc}
\frac{1}{4 \pi} \int_{-\pi}^{\pi}\left|\partial_{H} \log f_{H}(\lambda)\right|^{2} \mathrm{~d} \lambda & \frac{1}{2 \pi \sigma} \int_{-\pi}^{\pi} \partial_{H} \log f_{H}(\lambda) \mathrm{d} \lambda \\
\frac{1}{2 \pi \sigma} \int_{-\pi}^{\pi} \partial_{H} \log f_{H}(\lambda) \mathrm{d} \lambda & \frac{2}{\sigma^{2}}
\end{array}\right) .
$$

In particular, $\left(A_{n}, B_{n}\right)$ converges in law to a centered normal distribution with covariance

$$
\text { (3) } J(H)=\left(\begin{array}{cc}
2 & -\frac{1}{2 \pi} \int_{-\pi}^{\pi} \partial_{H} \log f_{H}(\lambda) \mathrm{d} \lambda \\
-\frac{1}{2 \pi} \int_{-\pi}^{\pi} \partial_{H} \log f_{H}(\lambda) \mathrm{d} \lambda & \frac{1}{4 \pi} \int_{-\pi}^{\pi}\left|\partial_{H} \log f_{H}(\lambda)\right|^{2} \mathrm{~d} \lambda
\end{array}\right) \text {. }
$$

ProOF. See [6].

3. The LAN property in high-frequency observation. Let $X_{n}$ be again the observed fractional Gaussian noise

$$
X_{n}=\left(\sigma B_{\Delta_{n}}^{H}, \sigma B_{2 \Delta_{n}}^{H}-\sigma B_{\Delta_{n}}^{H}, \sigma B_{3 \Delta_{n}}^{H}-\sigma B_{2 \Delta_{n}}^{H}, \ldots, \sigma B_{n \Delta_{n}}^{H}-\sigma B_{(n-1) \Delta_{n}}^{H}\right) .
$$

Here, we consider high-frequency asymptotics, which means $\Delta_{n} \rightarrow 0$ as $n \rightarrow \infty$. We suppose $\inf _{n} n \Delta_{n}>0$. The parameters to be estimated are still $H \in(0,1)$ and $\sigma>0$. As before, the distribution of

$$
\frac{1}{\sigma \Delta_{n}^{H}} X_{n}
$$

is stationary centered Gaussian and does not depend on $\sigma$ and $\Delta_{n}$ by the selfsimilarity property. Its covariance matrix is $T_{n}(H)$ defined in the previous section. Denote by $\ell_{n}(H, \sigma)$ the log-likelihood of $X_{n}$

$$
\begin{aligned}
\ell_{n}(H, \sigma)= & -\frac{n}{2} \log (2 \pi)-n H \log \Delta_{n}-n \log \sigma \\
& -\frac{1}{2} \log \left|T_{n}(H)\right|-\frac{1}{2 \sigma^{2} \Delta_{n}^{2 H}}\left\langle X_{n}, T_{n}(H)^{-1} X_{n}\right\rangle .
\end{aligned}
$$

Let

$$
\begin{aligned}
& A_{n}=\sqrt{n}\left\{\frac{1}{n \sigma^{2} \Delta_{n}^{2 H}}\left\langle X_{n}, T_{n}(H)^{-1} X_{n}\right\rangle-1\right\} \\
& B_{n}=\frac{1}{\sqrt{n}}\left\{\frac{1}{2} \partial_{H} \log \left|T_{n}(H)\right|+\frac{1}{2 \sigma^{2} \Delta_{n}^{2 H}}\left\langle X_{n}, \partial_{H}\left\{T_{n}(H)^{-1}\right\} X_{n}\right\rangle\right\} .
\end{aligned}
$$


We use the same notation as in the previous section because their distributions are the same. In particular, we have that $\left(A_{n}, B_{n}\right) \rightarrow(A, B)$ in law under $P_{(H, \sigma)}^{n}$ for a nondegenerate Gaussian random variable $(A, B)$ whose covariance is given by (3). In this setting, the score function is given by

$$
\nabla \ell_{n}=\left(\begin{array}{c}
\partial_{H} \ell_{n} \\
\partial_{\sigma} \ell_{n}
\end{array}\right)=\left(\begin{array}{c}
A_{n} \sqrt{n} \log \Delta_{n}-B_{n} \sqrt{n} \\
A_{n} \sqrt{n} \sigma^{-1}
\end{array}\right) .
$$

From this expression, we clearly see that the leading terms of $\partial_{H} \ell_{n}$ and $\partial_{\sigma} \ell_{n}$ are linearly dependent, which is exactly the reason why the Fisher matrix is singular when considering diagonal rate matrices as in [16]. Now, we state the main result of the paper.

THEOREM 3.1. Consider a sequence of matrices

$$
\varphi_{n}=\varphi_{n}(H, \sigma)=\frac{1}{\sqrt{n}}\left(\begin{array}{cc}
\alpha_{n} & \bar{\alpha}_{n} \\
\beta_{n} & \bar{\beta}_{n}
\end{array}\right)
$$

with the following properties:

1. $n\left|\varphi_{n}\right|=\alpha_{n} \bar{\beta}_{n}-\bar{\alpha}_{n} \beta_{n} \neq 0$.

2. $\alpha_{n} \rightarrow \alpha$ for some $\alpha \in \mathbb{R}$.

3. $\bar{\alpha}_{n} \rightarrow \bar{\alpha}$ for some $\bar{\alpha} \in \mathbb{R}$.

4. $\gamma_{n}:=\alpha_{n} \log \Delta_{n}+\beta_{n} \sigma^{-1} \rightarrow \gamma$ for some $\gamma \in \mathbb{R}$.

5. $\bar{\gamma}_{n}:=\bar{\alpha}_{n} \log \Delta_{n}+\bar{\beta}_{n} \sigma^{-1} \rightarrow \bar{\gamma}$ for some $\bar{\gamma} \in \mathbb{R}$.

6. $\alpha \bar{\gamma}-\bar{\alpha} \gamma \neq 0$.

Then the family $\left\{P_{(H, \sigma)}^{n} ;(H, \sigma) \in(0,1) \times(0, \infty)\right\}$ is LAN at any $(H, \sigma)$ for $\varphi_{n}(H, \sigma)$ defined previously and

$$
I(H, \sigma)=\left(\begin{array}{cc}
\gamma & -\alpha \\
\bar{\gamma} & -\bar{\alpha}
\end{array}\right) J(H)\left(\begin{array}{cc}
\gamma & \bar{\gamma} \\
-\alpha & -\bar{\alpha}
\end{array}\right),
$$

where $J(H)$ is defined by (3). The convergence of $r_{n}$ in (2) is uniform in $u$ on compact sets.

Proof. Let $\theta_{0}=\left(H_{0}, \sigma_{0}\right)^{*}, u \in \mathbb{R}^{2}$ and $\bar{B}\left(\theta_{0}, r\right)=\{\theta \in(0,1) \times(0, \infty)$; $\left.\left|\theta-\theta_{0}\right| \leq r\right\}$ for $r>0$. By Taylor's formula,

$$
\ell_{n}\left(\theta_{0}+\varphi_{n} u\right)-\ell_{n}\left(\theta_{0}\right)=\left\langle\varphi_{n}^{*} \nabla \ell_{n}\left(\theta_{0}\right), u\right\rangle+\frac{1}{2}\left\langle u, \varphi_{n}^{*} \nabla^{2} \ell_{n}\left(\theta_{0}\right) \varphi_{n} u\right\rangle+r_{n},
$$

where

$$
\left|r_{n}\right| \leq \frac{1}{6}\left|\varphi_{n} u\right||u|^{2} \max \left\{\left|\varphi_{n}^{*} \nabla^{3} \ell_{n}(\theta) \varphi_{n}\right| ; \theta \in \bar{B}\left(\theta_{0},\left|\varphi_{n} u\right|\right)\right\} .
$$

Step 1. Let us show that $\varphi_{n}^{*} \nabla \ell_{n}\left(\theta_{0}\right) \rightarrow \mathcal{N}\left(0, I\left(\theta_{0}\right)\right)$. Note that

$$
\varphi_{n}^{*} \nabla \ell_{n}=\left(\begin{array}{c}
A_{n} \gamma_{n}-B_{n} \alpha_{n} \\
A_{n} \bar{\gamma}_{n}-B_{n} \bar{\alpha}_{n}
\end{array}\right) .
$$


Therefore, it converges in law to

$$
\left(\begin{array}{l}
A \gamma-B \alpha \\
A \bar{\gamma}-B \bar{\alpha}
\end{array}\right)=\left(\begin{array}{ll}
\gamma & -\alpha \\
\bar{\gamma} & -\bar{\alpha}
\end{array}\right)\left(\begin{array}{l}
A \\
B
\end{array}\right)
$$

which is Gaussian.

Step 2. Here, we compute

$$
\varphi_{n}^{*} \nabla^{2} \ell_{n} \varphi_{n}
$$

Let

$$
\begin{aligned}
C_{n} & =\frac{1}{n \sigma^{2} \Delta_{n}^{2 H}}\left\langle X_{n}, T_{n}(H)^{-1} X_{n}\right\rangle, \\
D_{n} & =\frac{1}{n \sigma^{2} \Delta_{n}^{2 H}}\left\langle X_{n}, \partial_{H}\left\{T_{n}(H)^{-1}\right\} X_{n}\right\rangle, \\
E_{n} & =\frac{1}{n}\left\{\frac{1}{2} \partial_{H}^{2} \log \left|T_{n}(H)\right|+\frac{1}{2 \sigma^{2} \Delta_{n}^{2 H}}\left\langle X_{n}, \partial_{H}^{2}\left\{T_{n}(H)^{-1}\right\} X_{n}\right\rangle\right\} .
\end{aligned}
$$

Note that

$$
\partial_{H} \log \left|T_{n}(H)\right|=-\operatorname{tr}\left(\partial_{H}\left\{T_{n}(H)^{-1}\right\} T_{n}(H)\right)
$$

and so,

$$
\begin{aligned}
& \partial_{H}^{2} \log \left|T_{n}(H)\right| \\
& \quad=-\operatorname{tr}\left(\partial_{H}^{2}\left\{T_{n}(H)^{-1}\right\} T_{n}(H)\right)+\operatorname{tr}\left(T_{n}(H)^{-1} \partial_{H} T_{n}(H) T_{n}(H)^{-1} \partial_{H} T_{n}(H)\right) .
\end{aligned}
$$

Then the $(1,1)$ element of $\nabla^{2} \ell_{n} \varphi_{n}$ is

$$
\begin{aligned}
& \alpha_{n}\left(\log \Delta_{n} \partial_{H} A_{n}-\partial_{H} B_{n}\right)+\beta_{n} \frac{\partial_{H} A_{n}}{\sigma} \\
& \quad=\gamma_{n} \partial_{H} A_{n}-\alpha_{n} \partial_{H} B_{n} \\
& \quad=\gamma_{n} \sqrt{n}\left(-2 C_{n} \log \Delta_{n}+D_{n}\right)-\alpha_{n} \sqrt{n}\left(E_{n}-D_{n} \log \Delta_{n}\right)
\end{aligned}
$$

and the $(1,2)$ element is

$$
\begin{aligned}
& \alpha_{n} \frac{\partial_{H} A_{n}}{\sigma}+\beta_{n}\left(-\frac{A_{n}}{\sigma^{2}}+\frac{\partial_{\sigma} A_{n}}{\sigma}\right) \\
& =-2 \frac{\sqrt{n}}{\sigma} C_{n} \gamma_{n}+\frac{\sqrt{n}}{\sigma} D_{n} \alpha_{n}-\beta_{n} \frac{A_{n}}{\sigma^{2}} .
\end{aligned}
$$

It follows then that the $(1,1)$ element of $\varphi_{n}^{*} \nabla^{2} \ell_{n} \varphi_{n}$ is

$$
-2 C_{n} \gamma_{n}^{2}+2 D_{n} \gamma_{n} \alpha_{n}-\alpha_{n}^{2} E_{n}-\beta_{n}^{2} \frac{A_{n}}{\sigma^{2} \sqrt{n}} .
$$


From this, it is clear that the $(2,2)$ element of $\varphi_{n}^{*} \nabla^{2} \ell_{n} \varphi_{n}$ is

$$
-2 C_{n} \bar{\gamma}_{n}^{2}+2 D_{n} \bar{\gamma}_{n} \bar{\alpha}_{n}-\bar{\alpha}_{n}^{2} E_{n}-\bar{\beta}_{n}^{2} \frac{A_{n}}{\sigma^{2} \sqrt{n}} .
$$

Also it is already not difficult to see that the $(1,2)$ element of $\varphi_{n}^{*} \nabla^{2} \ell_{n} \varphi_{n}$ is

$$
-2 C_{n} \gamma_{n} \bar{\gamma}_{n}+D_{n}\left(\bar{\gamma}_{n} \alpha_{n}+\gamma_{n} \bar{\alpha}_{n}\right)-\alpha_{n} \bar{\alpha}_{n} E_{n}-\beta_{n} \bar{\beta}_{n} \frac{A_{n}}{\sigma^{2} \sqrt{n}} .
$$

It is clear that $n C_{n} \sim \chi^{2}(n)$ and so, $C_{n} \rightarrow 1$. We already know $A_{n} \rightarrow A$. By the conditions of $\varphi_{n}, \beta_{n}=O\left(\left|\log \Delta_{n}\right|\right)$ and $\bar{\beta}_{n}=O\left(\left|\log \Delta_{n}\right|\right)$. By the same argument as the proof of Lemma 3.5 of [6], we have

$$
D_{n} \rightarrow-\frac{1}{2 \pi} \int_{-\pi}^{\pi} \partial_{H} \log f_{H}(\lambda) \mathrm{d} \lambda=E[A B]
$$

and

$$
E_{n} \rightarrow \frac{1}{4 \pi} \int_{-\pi}^{\pi}\left|\partial_{H} \log f_{H}(\lambda)\right|^{2} \mathrm{~d} \lambda=E\left[B^{2}\right]
$$

Therefore,

$$
\begin{aligned}
& -\varphi_{n}^{*} \nabla^{2} \ell_{n} \varphi_{n} \\
& \quad \rightarrow\left(\begin{array}{cc}
2 \gamma^{2}-2 \gamma \alpha E[A B]+\alpha^{2} E\left[B^{2}\right] & 2 \gamma \bar{\gamma}-(\gamma \bar{\alpha}+\bar{\gamma} \alpha) E[A B]+\alpha \bar{\alpha} E\left[B^{2}\right] \\
2 \gamma \bar{\gamma}-(\gamma \bar{\alpha}+\bar{\gamma} \alpha) E[A B]+\alpha \bar{\alpha} E\left[B^{2}\right] & 2 \bar{\gamma}^{2}-2 \bar{\gamma} \bar{\alpha} E[A B]+\bar{\alpha}^{2} E\left[B^{2}\right]
\end{array}\right) .
\end{aligned}
$$

This coincides with $-I(H, \sigma)$ because $E\left[A^{2}\right]=2$.

Step 3. It remains to show $r_{n} \rightarrow 0$. From the computation in Step 2, we deduce that the tensor $-\varphi_{n}^{*} \nabla^{3} \ell_{n} \varphi_{n}$ consists of the vectors

$$
\begin{aligned}
& 2 \nabla C_{n} \gamma_{n}^{2}-2 \gamma_{n} \alpha_{n} \nabla D_{n}+\alpha_{n}^{2} \nabla E_{n}+\beta_{n}^{2} \frac{\nabla A_{n}}{\sigma^{2} \sqrt{n}}, \\
& 2 \nabla C_{n} \gamma_{n} \bar{\gamma}_{n}-\left(\gamma_{n} \bar{\alpha}_{n}+\bar{\gamma}_{n} \alpha_{n}\right) \nabla D_{n}+\alpha_{n} \bar{\alpha}_{n} \nabla E_{n}+\beta_{n} \bar{\beta}_{n} \frac{\nabla A_{n}}{\sigma^{2} \sqrt{n}}, \\
& 2 \nabla C_{n} \bar{\gamma}_{n}^{2}-2 \bar{\gamma}_{n} \bar{\alpha}_{n} \nabla D_{n}+\bar{\alpha}_{n}^{2} \nabla E_{n}+\bar{\beta}_{n}^{2} \frac{\nabla A_{n}}{\sigma^{2} \sqrt{n}} .
\end{aligned}
$$

By the same argument as the proof of Lemma 3.7 of [6], we have that there exists $\varepsilon>0$ such that $\nabla C_{n}, \nabla D_{n}$ and $\nabla E_{n}$ are of $O_{p}\left(n^{1 / 2-\varepsilon}\left|\log \Delta_{n}\right|\right)$ uniformly in $\theta \in$ $\bar{B}\left(\theta_{0}, \varepsilon\right)$. On the other hand,

$$
\begin{aligned}
\alpha_{n}^{2}+\bar{\alpha}_{n}^{2} & \rightarrow \alpha^{2}+\bar{\alpha}^{2}, \\
\frac{\beta_{n}^{2}+\bar{\beta}_{n}^{2}}{1+\sigma^{2}\left|\log \Delta_{n}\right|^{2}} & \rightarrow \alpha^{2}+\bar{\alpha}^{2},
\end{aligned}
$$


which implies that $\left|\varphi_{n} u\right|=O\left(\sqrt{|\log | \Delta_{n} \mid / n}\right)$. Since $\inf _{n} n \Delta_{n}>0$ by the assumption, we conclude that $r_{n} \rightarrow 0$ uniformly in $u$ on compact sets.

Several examples of rate matrices can be elicited:

1. A positive definite symmetric matrix for rate $\varphi_{n}$ is

$$
\begin{aligned}
\varphi_{n} & =\frac{1}{\sqrt{n}}\left(\begin{array}{cc}
-\frac{2}{\log \Delta_{n}} & \sigma \\
\sigma & -\sigma^{2} \log \Delta_{n}
\end{array}\right) \\
& =\frac{1}{\sqrt{n}}\left(\begin{array}{cc}
\cos \psi_{n} & \sin \psi_{n} \\
-\sin \psi_{n} & \cos \psi_{n}
\end{array}\right)\left(\begin{array}{cc}
\lambda_{n}^{+} & 0 \\
0 & \lambda_{n}^{-}
\end{array}\right)\left(\begin{array}{cc}
\cos \psi_{n} & -\sin \psi_{n} \\
\sin \psi_{n} & \cos \psi_{n}
\end{array}\right),
\end{aligned}
$$

where

$$
\begin{aligned}
\lambda_{n}^{ \pm} & =-\frac{1}{2}\left(\frac{2}{\log \Delta_{n}}+\sigma^{2} \log \Delta_{n} \pm \sqrt{\frac{4}{\left(\log \Delta_{n}\right)^{2}}+\sigma^{4}\left(\log \Delta_{n}\right)^{2}}\right), \\
\tan \psi_{n} & =-\frac{1}{2 \sigma}\left(\frac{2}{\log \Delta_{n}}-\sigma^{2} \log \Delta_{n}-\sqrt{\frac{4}{\left(\log \Delta_{n}\right)^{2}}+\sigma^{4}\left(\log \Delta_{n}\right)^{2}}\right),
\end{aligned}
$$

for which $\alpha=0, \bar{\alpha}=\sigma, \gamma=-1$ and $\bar{\gamma}=0$. There seems no simpler positive definite symmetric matrix satisfying the conditions of Theorem 3.1.

2. Let

$$
\varphi_{n}=\frac{1}{\sqrt{n}}\left(\begin{array}{cc}
\frac{\sigma \log \Delta_{n}}{1+\sigma^{2}\left(\log \Delta_{n}\right)^{2}} & -1 \\
\frac{1}{1+\sigma^{2}\left(\log \Delta_{n}\right)^{2}} & \sigma \log \Delta_{n}
\end{array}\right),
$$

for which the conditions of Theorem 3.1 are satisfied with $\alpha=\bar{\gamma}=0, \bar{\alpha}=-1$ and $\gamma=\sigma^{-1}$. Note that

$$
\varphi_{n}^{-1}=\sqrt{n}\left(\begin{array}{cc}
\lambda_{n}^{+} & 0 \\
0 & \lambda_{n}^{-}
\end{array}\right)\left(\begin{array}{cc}
\cos \psi_{n} & \sin \psi_{n} \\
-\sin \psi_{n} & \cos \psi_{n}
\end{array}\right),
$$

where

$$
\lambda_{n}^{+}=\sqrt{1+\sigma^{2}\left(\log \Delta_{n}\right)^{2}}, \quad \lambda_{n}^{-}=\frac{1}{\sqrt{1+\sigma^{2}\left(\log \Delta_{n}\right)^{2}}}
$$

and

$$
\cos \psi_{n}=\frac{\sigma \log \Delta_{n}}{\sqrt{1+\sigma^{2}\left(\log \Delta_{n}\right)^{2}}}, \quad \sin \psi_{n}=\frac{1}{\sqrt{1+\sigma^{2}\left(\log \Delta_{n}\right)^{2}}} .
$$

The above expression suggests that after the rotation of angle $\psi_{n}$ depending on $\Delta_{n}$ in a local parameter space, we have a familiar diagonal norming with, however, unfamiliar rates $\sqrt{n} \lambda_{n}^{ \pm}$. Note also that by setting formally $\Delta_{n}=1$, we recover Theorem 2.1. 
3. Another example is that

$$
\varphi_{n}=\frac{1}{\sqrt{n}}\left(\begin{array}{cc}
1 & 1 \\
\sigma\left(\gamma-\log \Delta_{n}\right) & \sigma\left(\bar{\gamma}-\log \Delta_{n}\right)
\end{array}\right)
$$

for $(\gamma, \bar{\gamma})$ with $\gamma \neq \bar{\gamma}$, for which $\alpha=\bar{\alpha}=1$.

4. Two other examples of rate matrices will be used in the following section, namely

$$
\varphi_{n}=\frac{1}{\sqrt{n}}\left(\begin{array}{cc}
1 & 0 \\
-\sigma \log \Delta_{n} & 1
\end{array}\right)
$$

which gives $\alpha=1, \bar{\alpha}=0, \gamma=0$ and $\bar{\gamma}=\sigma^{-1}$ and

$$
\varphi_{n}=\frac{1}{\sqrt{n}}\left(\begin{array}{cc}
\frac{1}{\log \Delta_{n}} & 1 \\
0 & -\sigma \log \Delta_{n}
\end{array}\right),
$$

which gives $\alpha=0, \bar{\alpha}=1, \gamma=1$ and $\bar{\gamma}=0$.

Remark that all the examples are nondiagonal rate matrix depending on the parameter $\sigma$.

\section{Efficient rates of estimation and optimality of ML estimators.}

4.1. The efficient estimation rate for $H$. As the rate matrix, let us take (8), which gives $\alpha=1, \bar{\alpha}=0, \gamma=0$ and $\bar{\gamma}=\sigma^{-1}$. It is worth mentioning that the rate matrix is nondiagonal and depends on the parameter $\sigma$. By Theorem II.11.2 of [14], the LAN property implies that for any regular estimator $\left(\hat{H}_{n}, \hat{\sigma}_{n}\right)$,

$$
\begin{aligned}
\liminf _{n \rightarrow \infty} E_{(H, \sigma)}^{n}\left[l\left(\varphi_{n}^{-1}\left(\begin{array}{c}
\hat{H}_{n}-H \\
\hat{\sigma}_{n}-\sigma
\end{array}\right)\right)\right] \\
\geq E\left[l\left(\left(\begin{array}{cc}
E\left[B^{2}\right] & -E[A B] / \sigma \\
-E[A B] / \sigma & 2 / \sigma^{2}
\end{array}\right)^{-1 / 2} N\right)\right]
\end{aligned}
$$

for any loss function $l$ satisfying the condition given in Theorem 1.1, where $N \sim$ $\mathcal{N}\left(0, I_{2}\right)$. Note that the matrix in the right-hand side coincides with $I(H, \sigma)$ in Theorem 2.1. Since

$$
\varphi_{n}^{-1}=\sqrt{n}\left(\begin{array}{cc}
1 & 0 \\
\sigma \log \Delta_{n} & 1
\end{array}\right)
$$

we obtain the asymptotic lower bound

$$
\liminf _{n \rightarrow \infty} n E_{(H, \sigma)}\left[\left(\hat{H}_{n}-H\right)^{2}\right] \geq \frac{2}{2 E\left[B^{2}\right]-E[A B]^{2}}
$$


by taking $l(x, y)=x^{2}$. This means that the efficient rate of estimation for $H$ is $\sqrt{n}$ when both $H$ and $\sigma$ are unknown. Note that when $\sigma$ is known, the efficient rate for $H$ is $\sqrt{n} \log \Delta_{n}$, which follows from, say, [16]. Although the singular LAN property of [16] seems to admit of a $\sqrt{n} \log \Delta_{n}$-consistent estimator even when $\sigma$ is unknown, the above lower bound implies that such an estimator, if any, cannot be regular. A possible relation to super-efficiency phenomena is remained as a topic for future research.

4.2. The efficient estimation rate for $\sigma$. As the rate matrix, let us take (9), which gives $\alpha=0, \bar{\alpha}=1, \gamma=1$ and $\bar{\gamma}=0$. It is worth repeating that the rate matrix is nondiagonal and depends on the parameter $\sigma$. Again by Theorem II.11.2 of [14], the LAN property implies that for any regular estimator $\left(\hat{H}_{n}, \hat{\sigma}_{n}\right)$,

$$
\liminf _{n \rightarrow \infty} E_{(H, \sigma)}^{n}\left[l\left(\varphi_{n}^{-1}\left(\begin{array}{c}
\hat{H}_{n}-H \\
\hat{\sigma}_{n}-\sigma
\end{array}\right)\right)\right] \geq E\left[l\left(\left(\begin{array}{cc}
2 & -E[A B] \\
-E[A B] & E\left[B^{2}\right]
\end{array}\right)^{-1 / 2} N\right)\right]
$$

for any loss function $l$ satisfying the condition given in Theorem 1.1, where $N \sim$ $\mathcal{N}\left(0, I_{2}\right)$. Since

$$
\varphi_{n}^{-1}=-\frac{\sqrt{n}}{\sigma}\left(\begin{array}{cc}
-\sigma \log \Delta_{n} & -1 \\
0 & \frac{1}{\log \Delta_{n}}
\end{array}\right)
$$

we obtain the asymptotic lower bound

$$
\liminf _{n \rightarrow \infty} \frac{n}{\sigma^{2}\left|\log \Delta_{n}\right|^{2}} E_{(H, \sigma)}\left[\left(\hat{\sigma}_{n}-\sigma\right)^{2}\right] \geq \frac{2}{2 E\left[B^{2}\right]-E[A B]^{2}}
$$

by taking $l(x, y)=y^{2}$. This means that the efficient rate of estimation for $\sigma$ is $\sqrt{n} /\left|\log \Delta_{n}\right|$ when both $H$ and $\sigma$ are unknown. Note that when $H$ is known, the efficient rate for $\sigma$ is $\sqrt{n}$, which follows from, say, [16]. Here applies a similar remark to the end of the previous subsection.

4.3. ML estimators. We prove, in this section, that a ML sequence of estimators $\left(\hat{H}_{n}, \hat{\sigma}_{n}\right)$ is asymptotically efficient in that the minimax bound in Theorem 1.1 is attained. By (4), it is given as a root of $A_{n}=B_{n}=0$ if any. Therefore,

$$
\begin{gathered}
\hat{\sigma}_{n}^{2}=\frac{1}{n \Delta_{n}^{2 \hat{H}_{n}}}\left\langle X_{n}, T_{n}\left(\hat{H}_{n}\right)^{-1} X_{n}\right\rangle, \\
\partial_{H} \log \left|T_{n}\left(\hat{H}_{n}\right)\right|+n \frac{\left\langle X_{n}, \partial_{H}\left\{T_{n}\left(\hat{H}_{n}\right)^{-1}\right\} X_{n}\right\rangle}{\left\langle X_{n}, T_{n}\left(\hat{H}_{n}\right)^{-1} X_{n}\right\rangle}=0 .
\end{gathered}
$$

The latter equation implies that, by the self-similarity property, the limit law of $\hat{H}_{n}$ is the same as in the large sample case treated in Section 2. In particular, $\hat{H}_{n}-H=$ 
$O_{p}\left(n^{-1 / 2}\right)$; see, for example, Dahlhaus [7]. On the other hand, the limit law of $\hat{\sigma}_{n}$ is different because

$$
\frac{\hat{\sigma}_{n}-\sigma}{\sigma} \approx \log \hat{\sigma}_{n}-\log \sigma \approx-\left(\hat{H}_{n}-H\right) \log \Delta_{n}
$$

as is easily seen. In particular, $\hat{\sigma}_{n}-\sigma=O_{p}\left(\left|\log \Delta_{n}\right| n^{-1 / 2}\right)$ and the joint law of

$$
\left(\sqrt{n}\left(\hat{H}_{n}-H\right), \frac{\sqrt{n}}{\log \Delta_{n}}\left(\hat{\sigma}_{n}-\sigma\right)\right)
$$

is degenerate to a one-dimensional distribution.

THEOREM 4.1. Let $\theta=(H, \sigma)^{*} \in(0,1) \times(0, \infty)$ and $\varphi_{n}=\varphi_{n}(H, \sigma)$ be a sequence of matrices satisfying the conditions of Theorem 3.1. Let $\hat{\theta}_{n}=\left(\hat{H}_{n}, \hat{\sigma}_{n}\right)^{*}$ be such a sequence that

$$
\varphi_{n}^{*} \nabla \ell_{n}\left(\hat{\theta}_{n}\right)=o_{p}(1), \quad \hat{\theta}_{n}-\theta=O_{p}\left(\left|\log \Delta_{n}\right|^{k} n^{-1 / 2}\right)
$$

for some $k \in \mathbb{N}$. Then

$$
\varphi_{n}^{-1}\left(\hat{\theta}_{n}-\theta\right) \rightarrow \mathcal{N}\left(0, I(H, \sigma)^{-1}\right)
$$

in law under $P_{(H, \sigma)}^{n}$ and

$$
\lim _{\delta \rightarrow \infty} \lim _{n \rightarrow \infty} \sup _{\left|\varphi_{n}^{-1}(\tau-\theta)\right| \leq \delta} E_{\tau}^{n}\left[l\left(\varphi_{n}^{-1}\left(\hat{\theta}_{n}-\tau\right)\right)\right]=\int_{\mathbb{R}^{2}} l\left(I(H, \sigma)^{-1 / 2} z\right) \phi(z) \mathrm{d} z
$$

for any bounded continuous function $l$ on $\mathbb{R}^{2}$, where $I(H, \sigma)$ is defined by $(5)$.

PROOF. By Taylor's formula,

$$
\varphi_{n}^{*} \nabla \ell_{n}\left(\hat{\theta}_{n}\right)=\varphi_{n}^{*} \nabla \ell_{n}(\theta)+\varphi_{n}^{*} \nabla^{2} \ell_{n}(\theta)\left(\hat{\theta}_{n}-\theta\right)+r_{n},
$$

where

$$
\left|r_{n}\right| \leq \frac{1}{2}\left|\hat{\theta}_{n}-\theta\right|\left|\varphi_{n}^{-1}\left(\hat{\theta}_{n}-\theta\right)\right| \max \left\{\left|\varphi_{n}^{*} \nabla^{3} \ell_{n}(\theta) \varphi_{n}\right| ; \theta \in \bar{B}\left(\theta_{0},\left|\hat{\theta}_{n}-\theta\right|\right)\right\} .
$$

Since $\alpha_{n} \bar{\beta}_{n}-\bar{\alpha}_{n} \beta_{n} \rightarrow \sigma(\alpha \bar{\gamma}-\bar{\alpha} \gamma)$, we have $\left\|\varphi_{n}^{-1}\right\|=O\left(\left|\log \Delta_{n}\right| n^{1 / 2}\right)$. Therefore, by the same argument as Step 3 in the proof of Theorem 3.1, we can show that $r_{n}=o_{p}(1)$. Then, by (6) and (7), we have

$$
\varphi_{n}^{-1}\left(\hat{\theta}_{n}-\theta\right) \approx-\left\{\varphi_{n}^{*} \nabla^{2} \ell_{n} \varphi_{n}\right\}^{-1} \varphi_{n}^{*} \nabla \ell_{n} \approx I(H, \sigma)^{-1}\left(\begin{array}{cc}
\gamma & -\alpha \\
\bar{\gamma} & -\bar{\alpha}
\end{array}\right)\left(\begin{array}{l}
A_{n} \\
B_{n}
\end{array}\right)
$$

From this expression, the first assertion of the theorem follows. Further, since

$$
\begin{aligned}
\log \frac{\mathrm{d} P_{\theta+\varphi_{n} u}^{n}}{\mathrm{~d} P_{\theta}^{n}} & \approx\left\langle\varphi_{n}^{*} \nabla \ell_{n}, u\right\rangle-\frac{1}{2}\langle u, I(H, \sigma) u\rangle \\
& \approx\left\langle\left(\begin{array}{c}
A_{n} \\
B_{n}
\end{array}\right),\left(\begin{array}{cc}
\gamma & \bar{\gamma} \\
-\alpha & -\bar{\alpha}
\end{array}\right) u\right\rangle-\frac{1}{2}\langle u, I(H, \sigma) u\rangle
\end{aligned}
$$


uniformly in $u$ on compact sets as we have seen in the proof of Theorem 3.1, the joint law of

$$
\left(\varphi_{n}^{-1}\left(\hat{\theta}_{n}-\theta\right), \log \frac{\mathrm{d} P_{\theta+\varphi_{n} u_{n}}^{n}}{\mathrm{~d} P_{\theta}^{n}}\right)
$$

under $P_{\theta}^{n}$ converges to a normal distribution with covariance

$$
E\left[I(H, \sigma)^{-1}\left(\begin{array}{cc}
\gamma & -\alpha \\
\bar{\gamma} & -\bar{\alpha}
\end{array}\right)\left(\begin{array}{l}
A \\
B
\end{array}\right)(A, B)\left(\begin{array}{cc}
\gamma & \bar{\gamma} \\
-\alpha & -\bar{\alpha}
\end{array}\right) u\right]=u
$$

for any sequence $u_{n}$ with $\lim _{n \rightarrow \infty} u_{n}=u$. Then, by Le Cam's third lemma,

$$
\varphi_{n}^{-1}\left(\hat{\theta}_{n}-\theta\right) \rightarrow \mathcal{N}\left(u, I(H, \sigma)^{-1}\right)
$$

in law under $P_{\theta+\varphi_{n} u_{n}}^{n}$, from which the second assertion follows.

4.4. Hypothesis testing. By the standard argument, as a corollary of Theorems 3.1 and 4.1, we have a limit theorem for the likelihood ratio test statistic $\Lambda_{n}$ for the hypothesis testing $\theta=\theta_{0}$ vs. $\theta \neq \theta_{0}$, that is,

$$
-2 \log \Lambda_{n} \approx-\left\langle\varphi_{n}^{-1}\left(\hat{\theta}_{n}-\theta_{0}\right), \varphi_{n}^{*} \nabla^{2} \ell_{n} \varphi \varphi_{n}^{-1}\left(\hat{\theta}_{n}-\theta_{0}\right)\right\rangle \rightarrow \chi^{2}(2)
$$

under the null hypothesis, where $\hat{\theta}_{n}$ is the ML estimator. On the other hand, for a composite null hypothesis, the usual argument fails due to the singularity of the asymptotic Fisher matrix. Still, we have the following result.

THEOREM 4.2. Consider the hypothesis testing $H=H_{0}$ vs. $H \neq H_{0}$ with $\sigma$ unknown. Let $\Lambda_{n}$ be the likelihood ratio statistic, that is,

$$
-\log \Lambda_{n}=\ell_{n}\left(\hat{\theta}_{n}\right)-\ell_{n}\left(\left(H_{0}, \tilde{\sigma}_{n}\left(H_{0}\right)\right)^{*}\right),
$$

where $\hat{\theta}_{n}$ is the ML estimator of $\theta=(H, \sigma)^{*}$ and $\tilde{\sigma}_{n}\left(H_{0}\right)$ is the ML estimator of $\sigma$ under the null hypothesis $H=H_{0}$. Then

$$
-2 \log \Lambda_{n} \rightarrow \chi^{2}(1)
$$

in law under the null hypothesis.

PROOF. Let $\varphi_{n}$ be a sequence of matrices satisfying the conditions of Theorem 3.1. Then, by (10) and $\left\langle\nabla \ell_{n}\left(\hat{\theta}_{n}\right),\left(\hat{\theta}_{n}-\theta\right)\right\rangle=\left\langle\varphi_{n}^{*} \nabla \ell_{n}\left(\hat{\theta}_{n}\right), \varphi_{n}^{-1}\left(\hat{\theta}_{n}-\theta\right)\right\rangle \approx 0$,

$$
\begin{aligned}
2\left(\ell_{n}\left(\hat{\theta}_{n}\right)-\ell_{n}(\theta)\right) & \\
& \approx\left\langle\varphi_{n}^{-1}\left(\hat{\theta}_{n}-\theta\right), I(H, \sigma) \varphi_{n}^{-1}\left(\hat{\theta}_{n}-\theta\right)\right\rangle \\
& \rightarrow\left\langle\left(\begin{array}{cc}
\gamma & \bar{\gamma} \\
-\alpha & -\bar{\alpha}
\end{array}\right)^{-1} J(H)^{-1}\left(\begin{array}{l}
A \\
B
\end{array}\right), I(H, \sigma)\left(\begin{array}{cc}
\gamma & \bar{\gamma} \\
-\alpha & -\bar{\alpha}
\end{array}\right)^{-1} J(H)^{-1}\left(\begin{array}{l}
A \\
B
\end{array}\right)\right\rangle \\
& =(A, B) J(H)^{-1}\left(\begin{array}{l}
A \\
B
\end{array}\right)
\end{aligned}
$$


and $(A, B)^{*} \sim J(H)^{1 / 2} N_{2}, N_{2} \sim \mathcal{N}\left(0, I_{2}\right)$. On the other hand,

$$
\frac{1}{n} \partial_{\sigma}^{2} \ell_{n}=-\frac{1}{\sigma^{2} \sqrt{n}}\left(A_{n}+2 \sqrt{n} C_{n}\right) \rightarrow-\frac{2}{\sigma^{2}}, \quad \sqrt{n}\left(\tilde{\sigma}_{n}(H)^{2}-\sigma^{2}\right)=\sigma^{2} A_{n}
$$

and so,

$$
2\left(\ell_{n}\left(\left(H, \tilde{\sigma}_{n}(H)\right)^{*}\right)-\ell_{n}(\theta)\right) \approx \frac{2}{\sigma^{2}}\left|\sqrt{n}\left(\tilde{\sigma}_{n}(H)-\sigma\right)\right|^{2} \rightarrow \frac{1}{2} A^{2} .
$$

Therefore,

$$
\begin{aligned}
-2 \log \Lambda_{n} & =2\left(\ell_{n}\left(\hat{\theta}_{n}\right)-\ell_{n}(\theta)\right)-2\left(\ell_{n}\left(\left(H, \tilde{\sigma}_{n}(H)\right)^{*}\right)-\ell_{n}(\theta)\right) \\
& \rightarrow(A, B)\left\{J(H)^{-1}-\frac{1}{2}\left(\begin{array}{ll}
1 & 0 \\
0 & 0
\end{array}\right)\right\}\left(\begin{array}{l}
A \\
B
\end{array}\right) \\
& =N_{2}^{*}\left\{I_{2}-\frac{1}{2} J(H)^{1 / 2}\left(\begin{array}{ll}
1 & 0 \\
0 & 0
\end{array}\right) J(H)^{1 / 2}\right\} N_{2} \sim \chi^{2}(1) .
\end{aligned}
$$

Here, we have used that the $(1,1)$ element of $J(H)$ is 2 , and so

$$
\begin{aligned}
\left|I_{2}-\frac{1}{2} J(H)^{1 / 2}\left(\begin{array}{ll}
1 & 0 \\
0 & 0
\end{array}\right) J(H)^{1 / 2}\right| & =0, \\
\operatorname{tr}\left(I_{2}-\frac{1}{2} J(H)^{1 / 2}\left(\begin{array}{ll}
1 & 0 \\
0 & 0
\end{array}\right) J(H)^{1 / 2}\right) & =1 .
\end{aligned}
$$

In order to argue asymptotic optimality of tests, it is conventional to compare their asymptotic powers under local alternatives. For the LAN family with nondiagonal rate matrices, it is not trivial at a glance which rate matrix we should take to define local alternatives. For our hypotheses $H=H_{0}$ vs. $H \neq H_{0}$, it would be natural to take (8) with unknown true value $\sigma=\sigma_{0}$ under which

$$
\left(\begin{array}{c}
H \\
\sigma
\end{array}\right)=\left(\begin{array}{l}
H_{0} \\
\sigma_{0}
\end{array}\right)+\varphi_{n}\left(\begin{array}{l}
u_{1} \\
u_{2}
\end{array}\right) \Leftrightarrow\left(\begin{array}{l}
u_{1} \\
u_{2}
\end{array}\right)=\sqrt{n}\left(\begin{array}{c}
H-H_{0} \\
\left(H-H_{0}\right) \sigma_{0} \log \Delta_{n}+\sigma-\sigma_{0}
\end{array}\right)
$$

so that the local null space is $\mathcal{N}:=\left\{\left(u_{1}, u_{2}\right)^{*} \mid u_{1}=0\right\}$. For other $\varphi_{n}$ than (8), the corresponding local null space depends on $n$ and so, seems difficult to treat. For $\varepsilon \in(0,1)$, we say a test $\psi_{n}$ is an asymptotically unbiased level $\varepsilon$ test at $\sigma_{0}$ if

$$
\limsup _{n \rightarrow \infty} E_{\theta_{0}+\varphi_{n} u}\left[\psi_{n}\right] \leq \min \left\{\varepsilon, \liminf _{n \rightarrow \infty} E_{\theta_{0}+\varphi_{n} \bar{u}}\left[\psi_{n}\right]\right\}
$$

for every $u \in \mathcal{N}$ and $\bar{u} \in \mathcal{N}^{c}$, where $\theta_{0}=\left(H_{0}, \sigma_{0}\right)^{*}$. We say a test $\psi_{n}$ is an asymptotically uniformly most powerful unbiased level $\varepsilon$ test at $\sigma_{0}$ if it is an asymptotically unbiased level $\varepsilon$ test at $\sigma_{0}$ and

$$
\liminf _{n \rightarrow \infty} E_{\theta_{0}+\varphi_{n} \bar{u}}\left[\psi_{n}\right] \geq \limsup _{n \rightarrow \infty} E_{\theta_{0}+\varphi_{n} \bar{u}}\left[\psi_{n}^{\prime}\right]
$$

for any $\bar{u} \in \mathcal{N}^{c}$ and any other asymptotically unbiased level $\varepsilon$ test $\psi_{n}^{\prime}$ at $\sigma_{0}$. 
THEOREM 4.3. Let $\Lambda_{n}$ be the likelihood ratio statistic of Theorem 4.2. The test

$$
\psi_{n}=1_{\left\{-2 \log \Lambda_{n} \geq z_{\varepsilon / 2}^{2}\right\}}, \quad 1-\Phi\left(z_{\varepsilon / 2}\right)=\varepsilon / 2
$$

is an asymptotically uniformly most powerful unbiased level $\varepsilon$ test at $\sigma_{0}$ for any $\sigma_{0}>0$.

ProOF. By Theorem 2 of Choi et al. [3],

$$
\limsup _{n \rightarrow \infty} E_{\theta_{0}+\varphi_{n} \bar{u}}\left[\psi_{n}^{\prime}\right] \leq P\left(\left|N_{1}+\sqrt{\kappa} \bar{u}_{1}\right| \geq z_{\varepsilon / 2}\right)
$$

for any $\bar{u}=\left(\bar{u}_{1}, \bar{u}_{2}\right)^{*} \in \mathcal{N}^{c}$ and any asymptotically unbiased level $\varepsilon$ test $\psi_{n}^{\prime}$ at $\sigma_{0}$, where $N_{1} \sim \mathcal{N}(0,1)$ and $\kappa$ is the effective information

$$
\kappa=\frac{\left|I\left(H_{0}, \sigma_{0}\right)\right|}{I\left(H_{0}, \sigma_{0}\right)_{2,2}}=\frac{\sigma^{2}}{2}\left|\left(\begin{array}{cc}
E\left[B^{2}\right] & -E[A B] / \sigma \\
-E[A B] / \sigma & 2 / \sigma^{2}
\end{array}\right)\right|=E\left[B^{2}\right]-\frac{1}{2} E[A B]^{2} .
$$

Here, we have used that $\alpha=1, \bar{\alpha}=0, \gamma=0$ and $\bar{\gamma}=\sigma^{-1}$ for (8). Actually, Choi et al. [3] assumed the LAN property with a diagonal rate matrix; however, their proof remains valid for nondiagonal cases as long as the local null space is of the form $\mathcal{N}=\left\{\left(u_{1}, u_{2}\right)^{*} \mid u_{1}=0\right\}$. On the other hand, by Theorem 4.2, the test $\psi_{n}$ is of asymptotic level $\varepsilon$. Further by (11), Le Cam's third lemma implies that $\left(A_{n}, B_{n}\right)^{*} \rightarrow \mathcal{N}\left(\mu(\bar{u}), J\left(H_{0}\right)\right)$ under $P_{\theta_{0}+\varphi_{n} \bar{u}}$, where

$$
\mu(\bar{u})=J\left(H_{0}\right)\left(\begin{array}{cc}
\gamma & \bar{\gamma} \\
-\alpha & -\bar{\alpha}
\end{array}\right) \bar{u}=J\left(H_{0}\right)\left(\begin{array}{c}
\bar{u}_{2} \sigma^{-1} \\
-\bar{u}_{1}
\end{array}\right)
$$

for any $\bar{u}=\left(\bar{u}_{1}, \bar{u}_{2}\right)^{*} \in \mathcal{N}^{c}$. Therefore, by the same computation as in (12), the limit law of $-2 \log \Lambda_{n}$ under $P_{\theta_{0}+\varphi_{n}} \bar{u}$ is

$$
\begin{aligned}
(\mu(\bar{u}) & \left.+J\left(H_{0}\right)^{1 / 2} N_{2}\right)^{*}\left\{J\left(H_{0}\right)^{-1}-\frac{1}{2}\left(\begin{array}{ll}
1 & 0 \\
0 & 0
\end{array}\right)\right\}\left(\mu(\bar{u})+J\left(H_{0}\right)^{1 / 2} N_{2}\right) \\
= & \kappa \bar{u}_{1}^{2}+2 \sqrt{\kappa} \bar{u}_{1} N+N^{2}=\left|N+\sqrt{\kappa} \bar{u}_{1}\right|^{2},
\end{aligned}
$$

where $N_{2} \sim \mathcal{N}\left(0, I_{2}\right), N$ is the first element of $Q N_{2}$ and $Q$ is an orthogonal matrix with

$$
Q^{*}\left(\begin{array}{ll}
1 & 0 \\
0 & 0
\end{array}\right) Q=I_{2}-\frac{1}{2} J(H)^{1 / 2}\left(\begin{array}{ll}
1 & 0 \\
0 & 0
\end{array}\right) J(H)^{1 / 2}
$$

Here, we have used (13), that

$$
J(H)-\frac{1}{2} J(H)\left(\begin{array}{ll}
1 & 0 \\
0 & 0
\end{array}\right) J(H)=\left(\begin{array}{ll}
0 & 0 \\
0 & \kappa
\end{array}\right)
$$

and that

$$
x^{2}=(x, y)\left(\begin{array}{ll}
1 & 0 \\
0 & 0
\end{array}\right)\left(\begin{array}{l}
x \\
y
\end{array}\right)
$$


for any $(x, y)^{*} \in \mathbb{R}^{2}$. This means that the upper bound of (14) is attained by $\psi_{n}$ and also that $\psi_{n}$ is asymptotically unbiased.

5. Concluding remarks. We have shown that the statistical experiment of the fGn under high-frequency observations enjoys the LAN property. The idea is the use of nondiagonal rate matrices. The self-similarity property is the key for this analysis. Therefore, an extension to the case where $\sigma$ is time-dependent is nontrivial. On the other hand, extensions to more general self-similar stationary Gaussian noises or stable Lévy noises under high-frequency observations are straightforward. For the latter, the singularity of the Fisher matrix was discussed in [1] and a weak LAN property was shown in [22], which exactly corresponds to the result of [16] for the fGn.

\section{REFERENCES}

[1] AÏT-SAhalia, Y. and JACOD, J. (2008). Fisher's information for discretely sampled Lévy processes. Econometrica 76 727-761. MR2433480

[2] Berzin, C. and León, J. R. (2008). Estimation in models driven by fractional Brownian motion. Ann. Inst. Henri Poincaré Probab. Stat. 44 191-213. MR2446320

[3] ChOI, S., HALl, W. J. and SCHICK, A. (1996). Asymptotically uniformly most powerful tests in parametric and semiparametric models. Ann. Statist. 24 841-861. MR1394992

[4] Clément, E. and Gloter, A. (2015). Local asymptotic mixed normality property for discretely observed stochastic differential equations driven by stable Lévy processes. Stochastic Process. Appl. 125 2316-2352. MR3322866

[5] Coeurjolly, J.-F. and Istas, J. (2001). Cramèr-Rao bounds for fractional Brownian motions. Statist. Probab. Lett. 53 435-447. MR1856169

[6] Cohen, S., Gamboa, F., Lacaux, C. and Loubes, J.-M. (2013). LAN property for some fractional type Brownian motion. ALEA Lat. Am. J. Probab. Math. Stat. 10 91-106. MR3083920

[7] Dahlhaus, R. (1989). Efficient parameter estimation for self-similar processes. Ann. Statist. 17 1749-1766. MR1026311

[8] Dahlhaus, R. (2006). Correction: "Efficient parameter estimation for self-similar processes" [Ann. Statist. 17 (1989), no. 4, 1749-1766; MR1026311]. Ann. Statist. 34 1045-1047. MR2283403

[9] Gloter, A. and JACOD, J. (2001). Diffusions with measurement errors. I. Local asymptotic normality. ESAIM Probab. Stat. 5 225-242. MR1875672

[10] Gloter, A. and JACOD, J. (2001). Diffusions with measurement errors. II. Optimal estimators. ESAIM Probab. Stat. 5 243-260. MR1875673

[11] Gobet, E. (2001). Local asymptotic mixed normality property for elliptic diffusion: A Malliavin calculus approach. Bernoulli 7 899-912. MR1873834

[12] Gobet, E. (2002). LAN property for ergodic diffusions with discrete observations. Ann. Inst. Henri Poincaré Probab. Stat. 38 711-737. MR1931584

[13] HÁJEK, J. (1972). Local asymptotic minimax and admissibility in estimation. 175-194. MR0400513

[14] Ibragimov, I. A. and HAS'Mins KII, R. Z. (1981). Statistical Estimation: Asymptotic Theory. Applications of Mathematics 16. Springer, New York. Translated from the Russian by Samuel Kotz. MR0620321

[15] Istas, J. and LANG, G. (1997). Quadratic variations and estimation of the local Hölder index of a Gaussian process. Ann. Inst. Henri Poincaré Probab. Stat. 33 407-436. MR1465796 
[16] KAWAI, R. (2013). Fisher information for fractional Brownian motion under high-frequency discrete sampling. Comm. Statist. Theory Methods 42 1628-1636. MR3041490

[17] KAWAI, R. (2013). Local asymptotic normality property for Ornstein-Uhlenbeck processes with jumps under discrete sampling. J. Theoret. Probab. 26 932-967. MR3119980

[18] KawaI, R. and Masuda, H. (2013). Local asymptotic normality for normal inverse Gaussian Lévy processes with high-frequency sampling. ESAIM Probab. Stat. 17 13-32. MR3002994

[19] Kutoyants, Y. A. (2004). Statistical Inference for Ergodic Diffusion Processes. Springer, London. MR2144185

[20] LE CAM, L. (1972). Limits of experiments. In Proceedings of the Sixth Berkeley Symposium on Mathematical Statistics and Probability (Univ. California, Berkeley, Calif., 1970/1971), Vol. I: Theory of Statistics 245-261. Univ. California Press, Berkeley, CA. MR0415819

[21] Lieberman, O., Rosemarin, R. and Rousseau, J. (2012). Asymptotic theory for maximum likelihood estimation of the memory parameter in stationary Gaussian processes. Econometric Theory 28 457-470. MR2913638

[22] Masuda, H. (2009). Joint estimation of discretely observed stable Lévy processes with symmetric Lévy density. J. Japan Statist. Soc. 39 49-75. MR2571802

[23] ReIss, M. (2011). Asymptotic equivalence for inference on the volatility from noisy observations. Ann. Statist. 39 772-802. MR2816338

[24] Rouss as, G. G. (1972). Contiguity of Probability Measures: Some Applications in Statistics. Cambridge Tracts in Mathematics and Mathematical Physics 63. Cambridge Univ. Press, London. MR0359099

[25] Taniguchi, M. and KaKiZawa, Y. (2000). Asymptotic Theory of Statistical Inference for Time Series. Springer, New York. MR1785484

[26] VAn Der VAart, A. W. (1998). Asymptotic Statistics. Cambridge Series in Statistical and Probabilistic Mathematics 3. Cambridge Univ. Press, Cambridge. MR1652247

Laboratoire Manceau de Mathématiques

LE MANS UNIVERSITÉ

Avenue Olivier Messiaen

72085 LE MANS CEDEX 9

FRANCE

E-MAIL: Alexandre.Brouste@univ-lemans.fr

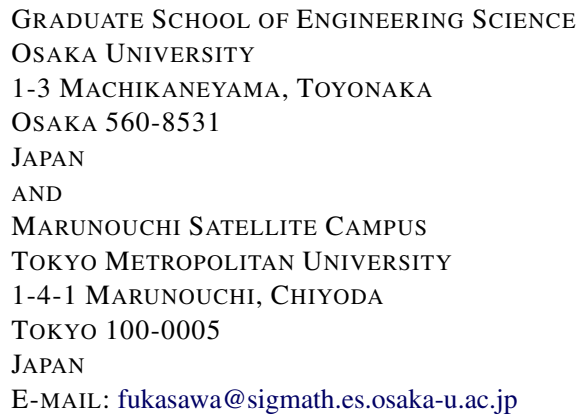

